\title{
Ex vivo imaging of human thyroid pathology using integrated optical coherence tomography and optical coherence microscopy
}

\author{
Chao Zhou \\ Massachusetts Institute of Technology \\ Department of Electrical Engineering and Computer Science \\ and \\ Research Laboratory of Electronics \\ 77 Massachusetts Avenue \\ Cambridge, Massachusetts 02139
}

\author{
Yihong Wang \\ Beth Israel Deaconess Medical Center \\ Harvard Medical School \\ Department of Pathology \\ 330 Brookline Avenue \\ Boston, Massachusetts 02215 \\ and \\ Montefiore Medical Center and Albert Einstein Medical \\ School \\ Department of Pathology \\ Bronx, New York 10461
}

\section{Aaron D. Aguirre}

Massachusetts Institute of Technology

Department of Electrical Engineering and Computer Science and

Research Laboratory of Electronics and

Harvard-MIT Division of Health Sciences and Technology

77 Massachusetts Avenue

Cambridge, Massachusetts 02139

\section{Tsung-Han Tsai}

Massachusetts Institute of Technology

Department of Electrical Engineering and Computer Science and

Research Laboratory of Electronics

77 Massachusetts Avenue

Cambridge, Massachusetts 02139

\section{David W. Cohen}

James L. Connolly

Beth Israel Deaconess Medical Center

Harvard Medical School

Department of Pathology

330 Brookline Avenue

Boston, Massachusetts 02215

\section{James G. Fujimoto}

Massachusetts Institute of Technology

Department of Electrical Engineering and Computer Science and

Research Laboratory of Electronics

77 Massachusetts Avenue

Cambridge, Massachusetts 02139

Address all correspondence to: James G. Fujimoto, Prof., Massachusetts Institute of Technology, Building 36-345, Cambridge, MA 02139. Tel: 617-253-8528; Fax: 617-253-9611; E-mail: jgfuji@mit.edu.

\begin{abstract}
We evaluate the feasibility of optical coherence tomography (OCT) and optical coherence microscopy (OCM) for imaging of benign and malignant thyroid lesions ex vivo using intrinsic optical contrast. 34 thyroid gland specimens are imaged from 17 patients, covering a spectrum of pathology ranging from normal thyroid to benign disease/neoplasms (multinodular colloid goiter, Hashimoto's thyroiditis, and follicular adenoma) and malignant thyroid tumors (papillary carcinoma and medullary carcinoma). Imaging is performed using an integrated OCT and OCM system, with $<4 \mu \mathrm{m}$ axial resolution (OCT and OCM), and $14 \mu \mathrm{m}$ (OCT) and $<2 \mu \mathrm{m}$ (OCM) transverse resolution. The system allows seamless switching between low and high magnifications in a way similar to traditional microscopy. Good correspondence is observed between optical images and histological sections. Characteristic features that suggest malignant lesions, such as complex papillary architecture, microfollicules, psammomatous calcifications, or replacement of normal follicular architecture with sheets/nests of tumor cells, can be identified from OCT and OCM images and are clearly differentiable from normal or benign thyroid tissues. With further development of needle-based imaging probes, OCT and OCM could be promising techniques to use for the screening of thyroid nodules and to improve the diagnostic specificity of fine needle aspiration evaluation.

() 2010 Society of Photo-Optical Instrumentation Engineers. [DOI: $10.1117 / 1.3306696]$
\end{abstract}

Keywords: optical coherence tomography; optical coherence microscopy; thyroid; pathology; cancer.

Paper 09283R received Aug. 7, 2009; revised manuscript received Nov. 4, 2009; accepted for publication Nov. 5, 2009; published online Feb. 22, 2010.

\section{Introduction}

Thyroid cancer is the most common malignancy of the endocrine system. ${ }^{1}$ Approximately 37,200 new cases and 1630 thyroid cancer deaths are expected in the United States in $2009 .{ }^{2}$ Various methods are used for the detection and screening of thyroid nodules for malignancy. These include clinical examination, various imaging methods, and ultrasound-guided fine needle aspiration (FNA). Thyroid cancer commonly presents as a cold (inactive) nodule on radioisotope scanning. Up to $40 \%$ of adults have a thyroid nodule detected by either palpation or ultrasound. ${ }^{3-6}$ Imaging techniques that can aid in the differentiation between benign and malignant thyroid nodules, which may require surgery, are of great interest. However, present imaging methods, including scintigraphy, ultrasound, $\mathrm{CT}$, and MRI, have only limited utility in the routine diagnostic assessment of thyroid nodules. ${ }^{7-9}$

$1083-3668 / 2010 / 15(1) / 016001 / 9 / \$ 25.00$ (C) 2010 SPIE 
Optical coherence tomography (OCT) is a promising technique for real-time, high resolution imaging of tissue morphology. ${ }^{10}$ Optical coherence microscopy (OCM) is an extension of OCT, which combines coherence gated detection with confocal microscopy to achieve cellular resolution imaging in the en face plane. ${ }^{11-15}$ By enhancing rejection of multiple scattered light, OCM can achieve better image contrast and greater imaging depth ${ }^{16}$ with lower numerical aperture ${ }^{12}$ compared with confocal microscopy. Integrated 3-D OCT and OCM have the additional advantage of enabling investigation of tissue structure at the architectural and cellular scale. 3-D OCT datasets enable cross-sectional and en face projection imaging, providing large field of view, while OCM provides high magnification, enabling cellular imaging. OCT has been previously investigated in many tissues, such as gastrointestinal tract, ${ }^{17-20}$ breast, ${ }^{21-23}$ urological organ, ${ }^{24,25}$ and thyroid. ${ }^{26}$ However, the transverse image resolution was limited to more than $10 \mu \mathrm{m}$. Combination of OCM with OCT overcomes this resolution limitation. Few studies using integrated OCT and OCM imaging have been performed, largely due to the lack of advanced OCM instrumentation.

In the present study, we employ an integrated OCT and OCM system to assess benign and malignant thyroid tissue ex vivo based on intrinsic optical contrast in freshly excised human thyroid specimens. Images of normal and pathologic tissue were compared with histological sections to recognize which histomorphologic features could be visualized using integrated OCT and OCM imaging. The results provide a basis for interpretation of future OCT and OCM images of the thyroid tissues, and suggest the possibility of future in vivo evaluation of thyroid pathology.

\section{Materials and Methods}

\subsection{Specimen Selection and Preparation}

The study protocol was approved by the institutional review boards at the Beth Israel Deaconess Medical Center (BIDMC) and the Massachusetts Institute of Technology (MIT). Informed consent was waived. Freshly excised thyroid specimens were selected based on the presence of pathology on gross examination. Normal and pathologic tissues were collected from each specimen without interfering with routine pathologic workup. Fresh tissue (typically measured $1 \times 1$ $\times 0.5 \mathrm{~cm}^{3}$ ) from surgical specimens that remained following processing for pathologic examination was collected for imaging and placed in RPMI medium 1640 (Invitrogen, Carlsbad, California) within $1 \mathrm{~h}$ after excision. Imaging was performed within $\sim 2$ to $6 \mathrm{~h}$ of excision. In total, 34 thyroid gland specimens were imaged from 17 patients (median age, 45 years; 12 females and 5 males). Ten benign and 13 malignant thyroid specimens were imaged. The specimens with benign diagnosis include goiters $(n=4)$, Hashimoto's thyroiditis $(n=3)$, and follicular adenoma $(n=3)$. The specimens with malignant diagnosis include papillary carcinoma, classic type $(n=6)$, papillary carcinoma, follicular variant $(n=6)$, and medullary carcinoma of the thyroid $(n=1)$. 11 matched normal thyroid specimens were obtained from total thyroidectomy specimens and evaluated as controls. Specimens were classified based on histological diagnosis by a pathologist with more than 30 years of experience.

\subsection{Integrated Optical Coherence Tomography and Optical Coherence Microscopy System}

A portable prototype imaging system integrating 3-D OCT and OCM was employed for the study. A detail description of the system design can be found in Ref. 27. The system uses a compact, spectrally broadened, femtosecond Nd:glass laser light source, which provides $>200 \mathrm{~nm}$ bandwidth centered at $1060 \mathrm{~nm}$. The output from the laser was split equally into the OCT and OCM subsystems. The OCT subsystem had a $<4 \mu \mathrm{m}$ axial resolution and $14 \mu \mathrm{m}$ transverse resolution. The axial resolution corresponds to optical image slices thinner than traditional histological sections. A pair of high speed scanning galvonometers enables the beam to be scanned in two dimensions, allowing 640 cross-sectional OCT images to be acquired with $1344 \times 1000$ (transverse $\times$ axial) pixels at $1 \mathrm{frame} / \mathrm{sec}$. This results in a 3-D dataset covering a volume of $3 \times 1.5 \times 1.3 \mathrm{~mm}^{3}(x \times y \times z)$. The OCT signal was demodulated and logarithmically compressed using an analog circuit before analog-to-digital conversion.

The OCM subsystem shares the same sample arm optics as the OCT subunit, except for the objective lens $(40 \times$, Zeiss Achroplan), which was turret mounted to allow rapid interchange between high (OCM) and low (OCT) magnifications. The aperture of the objective is not fully filled, and the resulting confocal parameter is $\sim 30 \mu \mathrm{m}$. The transverse image resolution for OCM was $<2 \mu \mathrm{m}$. A separate reference arm utilizes dispersion-compensating optics and enables an axial resolution of $<4 \mu \mathrm{m}$ in tissue. The penetration depth of the OCT and OCM system depends on the scattering properties of the sample, and over 500- and 300- $\mu \mathrm{m}$ imaging depth (respectively) was obtained in human thyroid tissues. A high speed, broadband electro-optic phase modulator was used, enabling rapid image acquisition with raster scanning and demodulation over a $400 \times 400 \mu \mathrm{m}^{2}$ field $(500 \times 750$ pixels $)$ at 2 frames/sec. A detection sensitivity of $-98 \mathrm{~dB}$ was achieved with $\sim 10 \mathrm{~mW}$ of incident power.

\subsection{Imaging Procedures and Histological Preparation}

A thin coverslip was gently placed on the specimen to create a flat surface and reduce optical aberration. The light pressure applied to the specimen is not expected to influence tissue morphology and histological comparison with OCT/OCM imaging. 3-D OCT images were first acquired. En face OCM data were then collected within the same imaging area to ensure good coregistration. A 3-D OCM dataset was acquired on some specimens by scanning the sample stage in the axial direction at $5 \mu \mathrm{m} / \mathrm{s}$. A gross photograph was taken before the specimen was marked with black and red ink spots on the imaging surface to indicate orientation. The specimen was formalin fixed and sent for histological processing. Sections were cut in en face planes to allow coregistration to both the en face OCT and OCM images. Slides were stained with hematoxylin and eosin (H\&E), and photomicrographs were digitally acquired using a standard microscope (Olympus BX40).

\subsection{Data Analysis}

Surfaces of the 2-D OCT cross-sectional images were detected and flattened in postprocessing to allow en face image planes to be viewed at constant depth. En face slices of OCT images $\left(3 \times 1.5 \mathrm{~mm}^{2}\right)$ were reconstructed from the $3-\mathrm{D}$ 


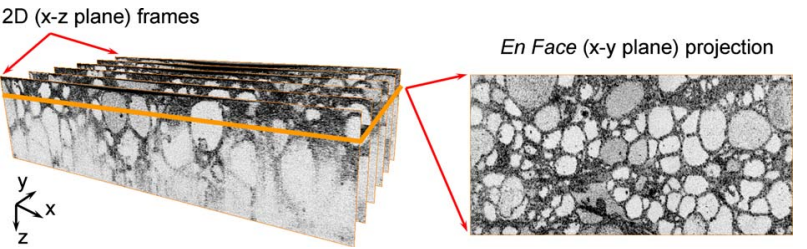

Fig. 1 En face OCT image (right) was constructed from the 3-D volumetric dataset (left), consisting of 640 2-D cross-sectional OCT images.

datasets by averaging over $10 \mu \mathrm{m}$ in the axial direction to reduce speckle noise (Fig. 1). The en face OCM data were processed by digital demodulation, pixel resampling, spatial filtering $(3 \times 3$ triangular kernel), and square-root compression of the signal. The OCT and OCM images are contrast adjusted and displayed with an inverse grayscale color map, where black represents increased reflectivity. In this retrospective study, the entire en face OCT and OCM database and histology slides were first reviewed, and representative normal and pathologic specimens were selected for further evaluation. The registration procedure works well to identify the region of interest for comparison to histology. However, direct one-to-one image registration to histology remain challenging because the exact orientation of the histological section is difficult to control. The generation of volumetric OCT and OCM data provide more comprehensive information than individual histological sections. Selection was based on several factors, including the degree to which the images match histology, and the degree to which identified image features accurately represented the larger dataset. Representative photomicrographs of histological sections were then made with a best-effort attempt to provide comparison with the en face OCT and OCM images. The features used for comparison included the size and shapes of follicles, papillae, patterns of stromal tissue, calcifications, vascular features, and cellular distribution. This protocol ensured that comparable features

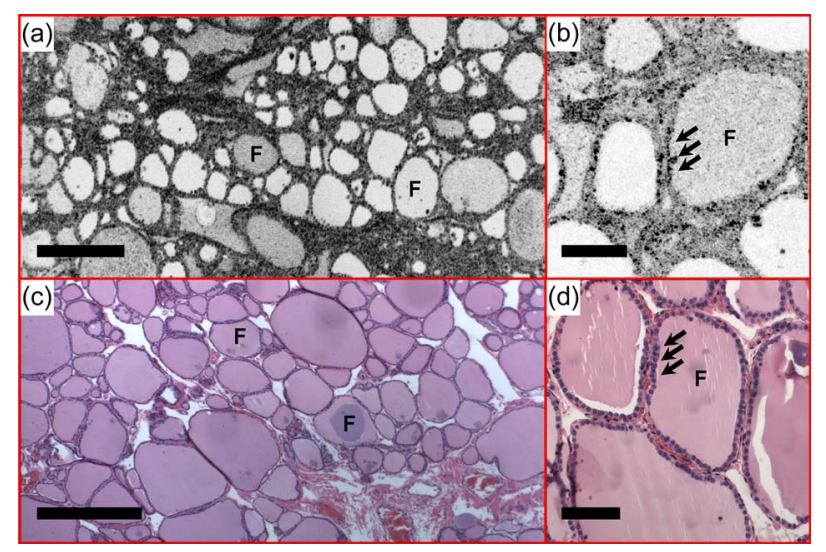

Fig. 2 Normal thyroid demonstrates well-organized round to oval thyroid follicles (F). Colloid with various densities was observed under (a) en face OCT and (b) OCM, obtained about 100 and $50 \mu \mathrm{m}$ below the tissue surface respectively, as different signal strength. The follicles are lined by a single layer of epithelium (arrows), which are clearly seen in the OCM image (b). (c) and (d) are corresponding HE slides (4 and $20 \times$, respectively). Scale bars, $500 \mu \mathrm{m}$ in (a) and (c), and $100 \mu \mathrm{m}$ in (b) and (d).

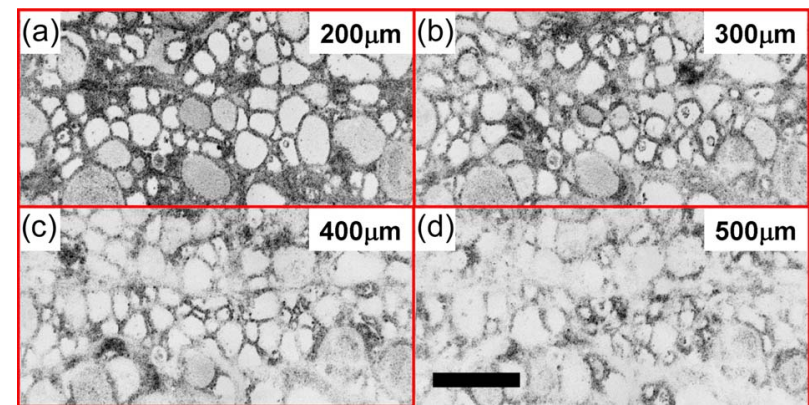

Fig. 3 En face OCT images of normal thyroid from various depths. Scale bar, $500 \mu \mathrm{m}$.

were matched on OCT/OCM images and histological sections.

\section{Results}

\subsection{Normal Thyroid}

Figure 2 shows representative images of normal thyroid. Round or oval thyroid follicles, ranging from 50 to $500 \mu \mathrm{m}$ in diameter, are observed and appear well organized in the en face OCT image [Fig. 2(a)]. A follicle lined by a single layer of epithelium can be clearly seen in the OCM image [Fig. 2(b)]. The corresponding histology [Figs. 2(c) and 2(d)] matches well with the OCT and OCM images. En face OCT and OCM images obtained from various tissue depths were displayed in Figs. 3 and 4, respectively. Image quality decreases in both OCT and OCM as the imaging depth increases due to the influence of multiple scattering. However, the follicle shape and the lining of a single layer of epithelium can still be appreciated from images obtained in deep tissue (about 400 to $500 \mu \mathrm{m}$ in OCT and 250 to $300 \mu \mathrm{m}$ in OCM). A 3-D OCM reconstruction of the normal follicles can be found in the supplemental materials (Video 1).

\subsection{Benign Diseases}

\subsubsection{Multinodular colloid goiter}

Figure 5 shows an example of a multinodular colloid goiter. Thyroid follicles with variable sizes and shapes are observed from the en face OCT image [Fig. 5(a)]. Increased cellularity and reduced (paler) amount of colloid in the follicle are observed. Large blood vessels can be seen from the en face OCT and corresponding histology [Figs. 5(a) and 5(c)]. OCM images $[$ Fig. $5(\mathrm{~b})]$ provide an enlarged view of the atrophy fol-

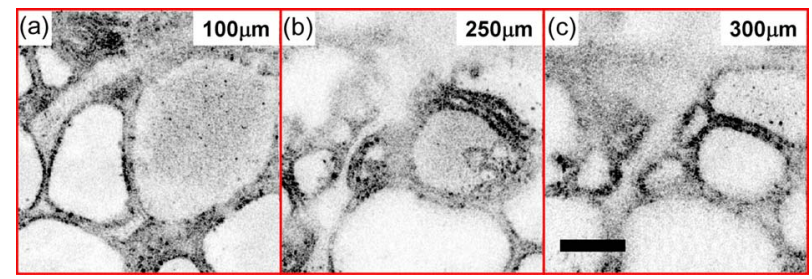

Fig. 4 En face OCM images of normal thyroid from various depths. Scale bar, $100 \mu \mathrm{m}$. A 3-D OCM reconstruction of the normal follicles can be found in Video 1. 


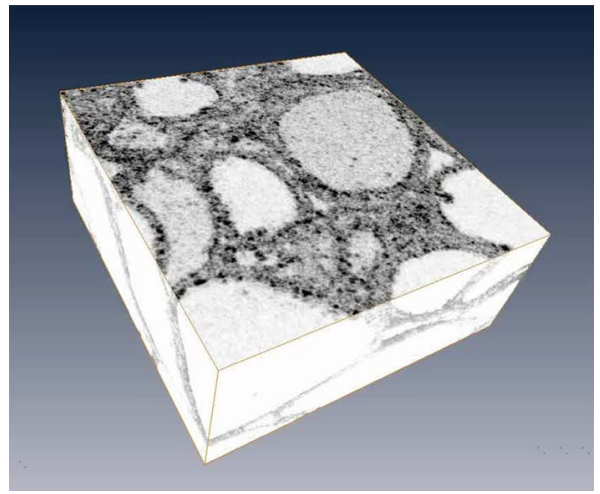

Video 1 Three-dimensional OCM reconstruction of normal human thyroid showing round and oval follicles. Volume size is $400 \times 400$ $\times 300 \mu \mathrm{m}$ (QuickTime, 1.5 MB).

[URL: http://dx.doi.org/10.1117/1.3306696.1].

licles and are consistent with histological observations [Fig. 5(d)].

\subsubsection{Hashimoto's thyroiditis}

Hashimoto's thyroiditis is an autoimmune disease characterized by extensive lymphocytic infiltration with germinal center formation and Hürthle cell change. Figure 6 is an example of Hashimoto's thyroiditis. In addition to the observation of enlarged and distorted follicles from the en face OCT and OCM images, we observe marked increased interfollicular cellular density with nodule configuration and central palor, which corresponds well with interstitial lymphocytic infiltrate and germinal center formation.

\subsubsection{Fibrous variant of Hashimoto's thyroiditis}

Images in Fig. 7 are characterized by extensive dense fibrosis, which is consistent with a fibrous variant of Hashimoto's thyroiditis. Although the lobular architecture of the gland is maintained, follicular atrophy is apparent. The colloid in some

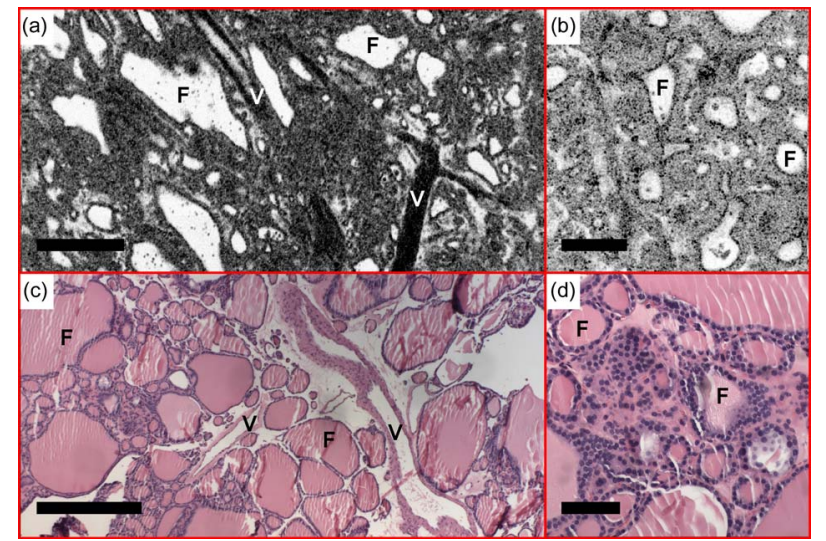

Fig. 5 (a) En face OCT and (b) OCM, obtained about 60 and $50 \mu \mathrm{m}$ below the tissue surface, respectively, demonstrate thyroid follicles (F) with variable sizes and shapes consistent with multinodular colloid goiter. Increased cellularity, reduced amount of colloid, and large blood vessels (V) were observed. (c) and (d) are corresponding HE slides (4 and 20×, respectively). Scale bars, $500 \mu \mathrm{m}$ in (a) and (c), and $100 \mu \mathrm{m}$ in (b) and (d).

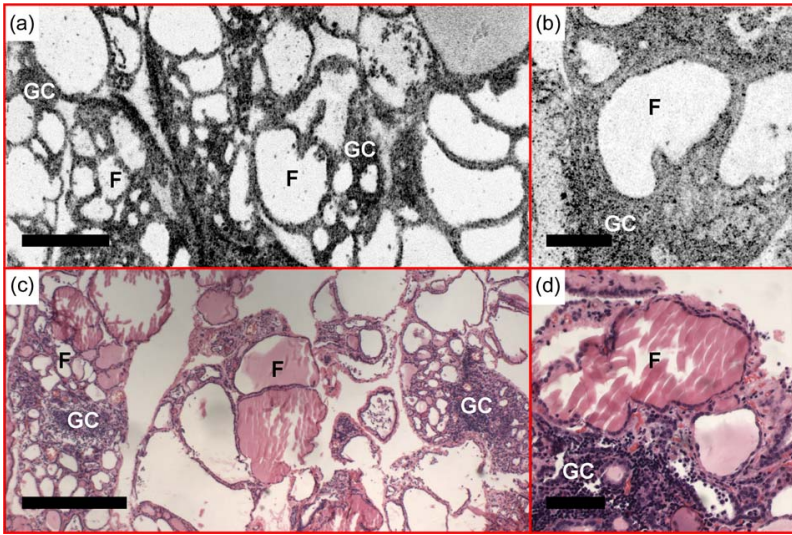

Fig. 6 Example of Hashimoto's thyroiditis. (a) En face OCT and (b) OCM, obtained about 230 and $50 \mu \mathrm{m}$ below the tissue surface, respectively, demonstrate enlarged and distorted follicles (F), increased interfollicular cellular density, interstitial lymphocytic infiltration, and germinal center (GC) formation. (c) and (d) are corresponding $\mathrm{HE}$ slides (4 and 20×, respectively). Scale bars, $500 \mu \mathrm{m}$ in (a) and (c), and $100 \mu \mathrm{m}$ in (b) and (d).

of the follicles demonstrates features with concentrated circles, which is consistent with the existence of hard colloid [Figs. 7(b) and 7(d)].

\subsubsection{Follicular adenoma}

Follicular adenoma is a benign encapsulated tumor with follicular differentiation throughout the nodule. Figure 8 shows a representative case. As can be seen from the OCT and OCM images, the follicle size varies significantly, ranging from 40 to $800 \mu \mathrm{m}$ in diameter. This observation corresponds to findings from histological sections and is also confirmed by images from other specimens.

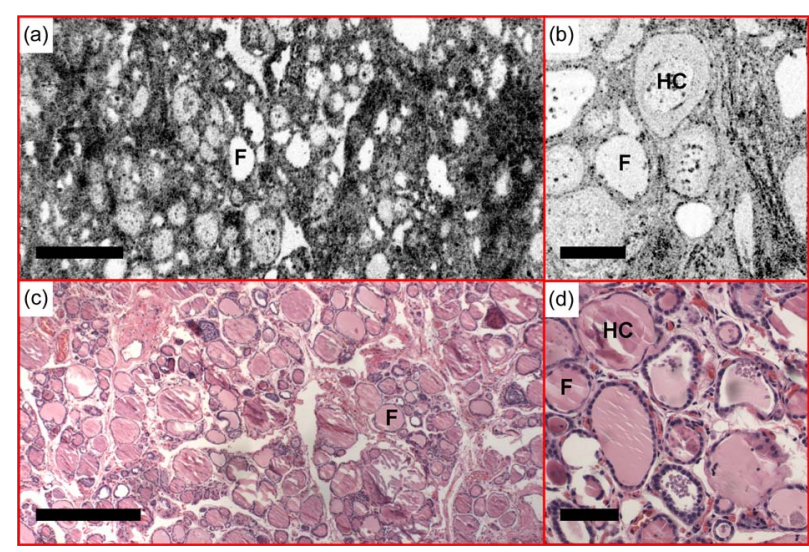

Fig. 7 Fibrous variant of Hashimoto's thyroiditis was observed with (a) en face OCT and (b) OCM, obtained about 160 and $50 \mu \mathrm{m}$ below the tissue surface, respectively. Existence of hard colloid (HC) was observed under (b) OCM, featuring concentrated circles. (c) and (d) are corresponding HE slides (4 and 20×, respectively). Scale bars, $500 \mu \mathrm{m}$ in (a) and (c), and $100 \mu \mathrm{m}$ in (b) and (d). 


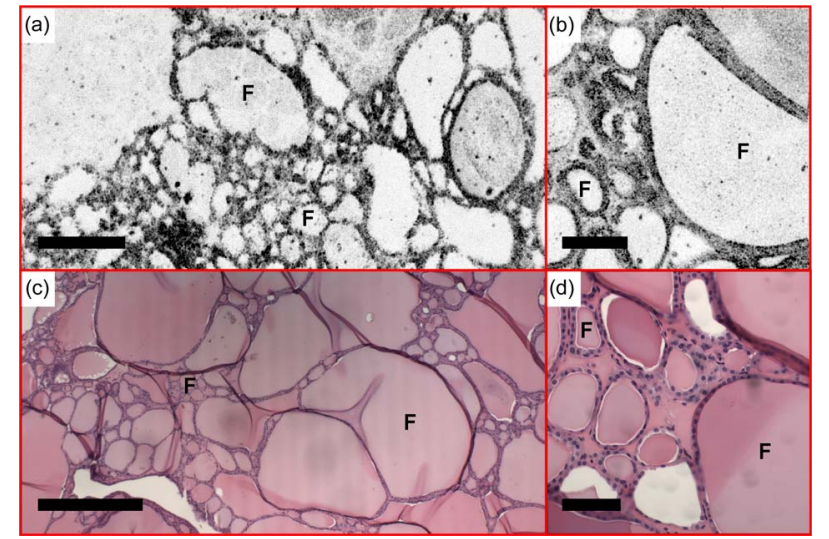

Fig. 8 (a) En face OCT and (b) OCM of follicular adenoma, obtained about 500 and $50 \mu \mathrm{m}$ below the tissue surface, respectively, show follicles (F) with significantly varying sizes. (c) and (d) are corresponding HE slides (4 and 20×, respectively). Scale bars, $500 \mu \mathrm{m}$ in (a) and (c), and $100 \mu \mathrm{m}$ in (b) and (d).

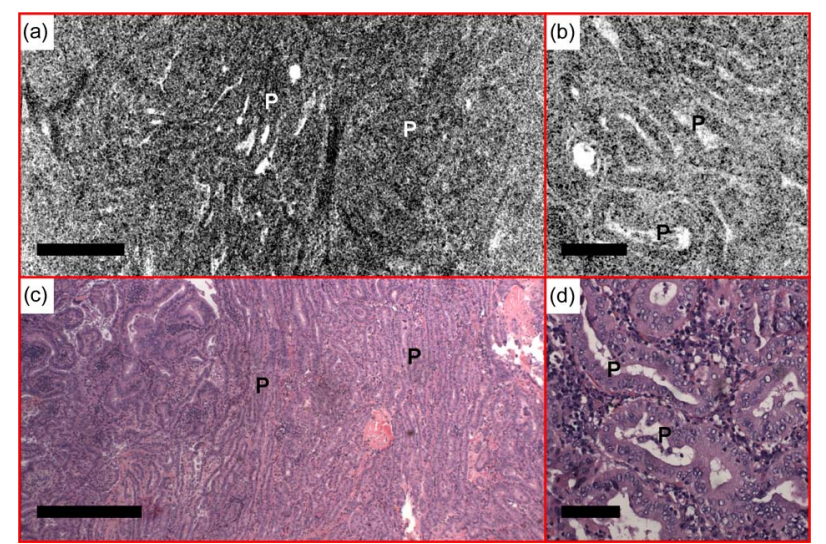

Fig. 9 Normal follicles are absent from the (a) en face OCT and (b) OCM images, obtained about 110 and $50 \mu \mathrm{m}$ below the tissue surface, respectively, in a case of classic-type papillary carcinoma. The thyroid is replaced by complex papillae (P), showing irregular papillary fronds and complex branching features. (c) and (d) are corresponding HE slides (4 and 20x, respectively). Scale bars, $500 \mu \mathrm{m}$ in (a) and (c), and $100 \mu \mathrm{m}$ in (b) and (d).

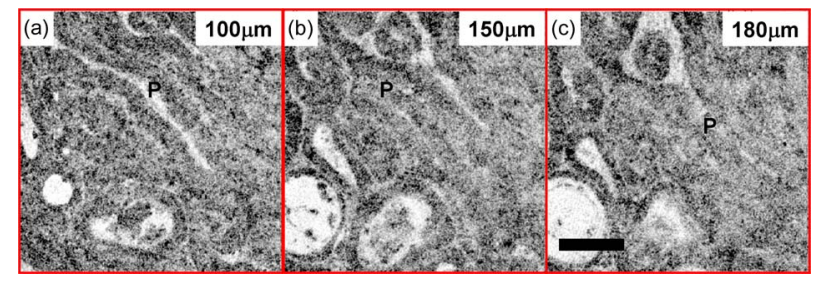

Fig. 10 En face OCM images of papillary carcinoma from various depths. The papillae structure can be visible from $180 \mu \mathrm{m}$ below the tissue surface. Scale bar, $100 \mu \mathrm{m}$. A 3-D OCM reconstruction of the papillae is provided can be found in Video 2 .

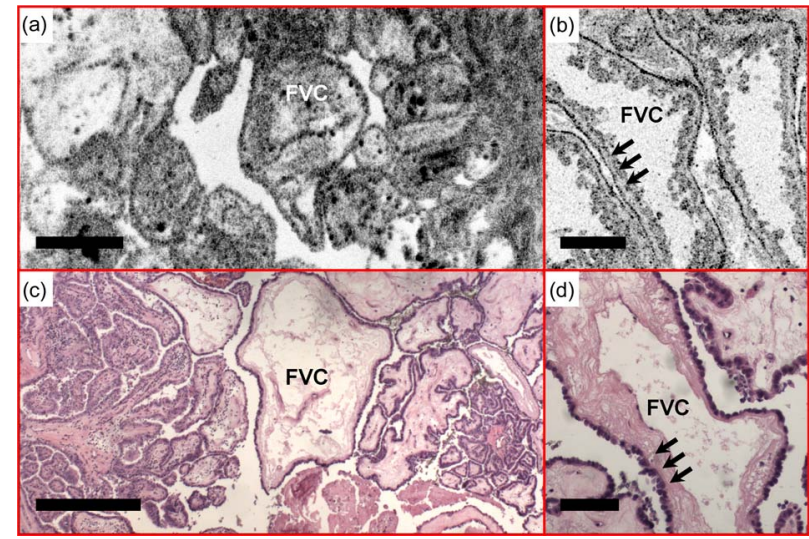

Fig. 11 Fibrovascular cores (FVCs) of the papillae, another representative feature of classic-type papillary carcinoma, are clearly identified in the (a) en face OCT and (b) OCM images obtained about 100 and $50 \mu \mathrm{m}$ below the tissue surface, respectively. A single layer of epithelium lines the follicles (arrows), with underlying dense fibrosis. (c) and (d) are corresponding HE slides (4 and 20×, respectively). Scale bars, $500 \mu \mathrm{m}$ in (a) and (c), and $100 \mu \mathrm{m}$ in (b) and (d).

\subsection{Malignant Diseases}

\subsubsection{Papillary carcinoma, classic type}

Figures 9-12 show several examples of papillary carcinoma, which is the most common type of thyroid cancer in the United States (75 to 80\%). ${ }^{28}$ As shown in Figs. 9(a) and 9(b), normal follicles are absent in classic-type papillary carcinoma. Instead, the thyroid is replaced by complex papillae, as confirmed by histological sections [Fig. 9(c)]. Detailed papillary structures can be appreciated from the OCM image shown in Fig. 9(b). En face OCM images from various depths are shown in Fig. 10. Even though tissue scattering is higher in papillary carcinoma specimens, the papillae structures from as deep as $180 \mu \mathrm{m}$ below the tissue surface can be visualized. A 3-D OCM reconstruction of the papillae can be viewed in the supplemental materials (Video 2), showing complex branching features. Another representative feature, the fibrovascular core of the papillae, is clearly seen in a different

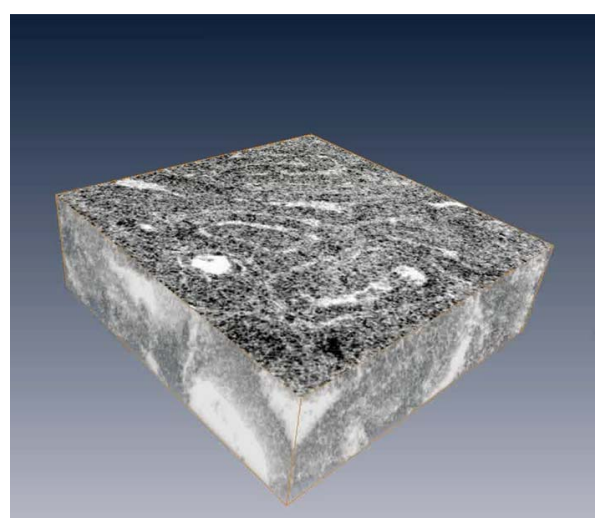

Video 2 Three-dimensional OCM reconstruction of human thyroid with papillary carcinoma. Normal follicles of the thyroid are replaced by complex papillae, showing irregular papillary fronds and complex branching features. Volume size is $400 \times 400 \times 130 \mu \mathrm{m}$ (QuickTime, 1.4 MB). [URL: http://dx.doi.org/10.1117/1.3306696.2]. 


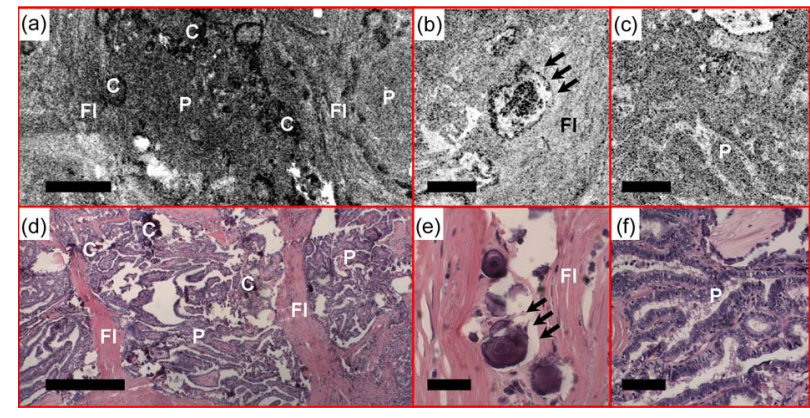

Fig. 12 Another case of classic-type papillary carcinoma, demonstrating features such as calcifications $(C)$, clusters of papillae $(P)$, and dense fibrosis ( $\mathrm{FI})$ in the (a) en face OCT and (b) and (c) OCM images obtained about $50 \mu \mathrm{m}$ below the tissue surface. Psammoma bodies (arrows) can be clearly identified by (b) OCM. (d), (e), and (f) are corresponding $\mathrm{HE}$ slides (4 and $20 \times$, respectively). Scale bars, $500 \mu \mathrm{m}$ in (a) and (d), and $100 \mu \mathrm{m}$ in (b), (c), (e), and (f).

specimen (Fig. 11). A single layer of epithelium lined the follicles with underlying stromal fibrosis. The corresponding histological sections [Figs. 11(c) and 11(d)] match well with the OCT and OCM images.

Figure 12 shows another example of classic-type papillary carcinoma. Calcifications and dense fibrosis separating the papillary fronds are clearly identified in the en face OCT image [Fig. 12(a)]. The OCM image in Fig. 12(b) demonstrates Psammoma bodies, which are a pattern of calcification commonly associated with papillary carcinoma. Overall, the OCT and OCM images enable visualization of diagnostically significant features for classic-type papillary carcinoma.

\subsubsection{Follicular variant of papillary carcinoma}

Figures 13-15 show examples of the follicular variant of papillary carcinoma. The nodular thyroid shows a homogenous microfollicullar pattern [Figs. 13(a) and 13(c)]. Details of the

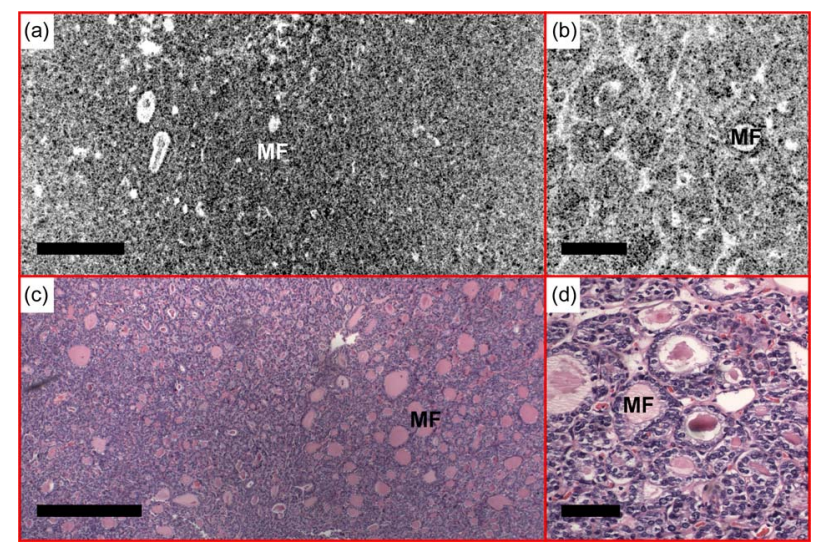

Fig. 13 A representative case of the follicular variant of papillary carcinoma. (a) En face OCT showed a homogeneous microfollicular (MF) pattern, where the details can be seen under (b) OCM. The en face OCT and OCM images were obtained about 200 and $50 \mu \mathrm{m}$ below the tissue surface, respectively. The size of the microfollicles is approximately $50 \mu \mathrm{m}$, consistent with the HE histology in (c) and (d), 4 and $20 \times$, respectively. Scale bars, $500 \mu \mathrm{m}$ in (a) and (c), and $100 \mu \mathrm{m}$ in (b) and (d).

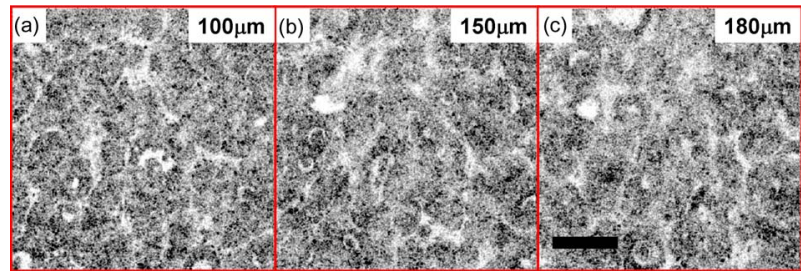

Fig. 14 En face OCM images of follicular variant of papillary carcinoma from various depths. The papillae structure can be visible from $180 \mu \mathrm{m}$ below the tissue surface. Scale bar, $100 \mu \mathrm{m}$. A 3-D OCM reconstruction of the microfollicles can be found in Video 3.

microfollicles can be seen in the OCM image. The size of the microfollicles is $\sim 50 \mu \mathrm{m}$, consistent with histology. Figure 14 shows en face OCM images from various depths. Microfollicles can be clearly identified about $180 \mu \mathrm{m}$ below the tissue surface. A 3-D OCM reconstruction of the microfollicles is provided in the supplemental materials (Video 3). Figure 15 shows another case where the en face OCT image was performed at the interface between the tumor and normal tissue [Fig. 15(a)]. The tumor on the left can be clearly distinguished as densely packed microfollicles, separated from the adjacent normal thyroid tissue at the lower right corner by a dense fibrous capsule. OCM reveals details of microfollicles [Fig. 15(b)] and normal follicles [Fig. 15(c)] from the same specimen, consistent with the histology. Although the resolution of OCM is not at the level for cytologic diagnosis of this disease, the clear observation of the microfollicular pattern provides valuable information suggesting the follicular variant of papillary carcinoma, which is currently not available in any of the in vivo imaging modality used in clinical practice.

\subsubsection{Medullary carcinoma}

Medullary carcinoma is a less common malignant tumor in the thyroid, representing about 3 to $5 \%$ of thyroid carcinomas. $^{28}$ The malignant cells are derived from parafollicular cells, or $\mathrm{C}$ cells, that normally secrete calcitonin. In

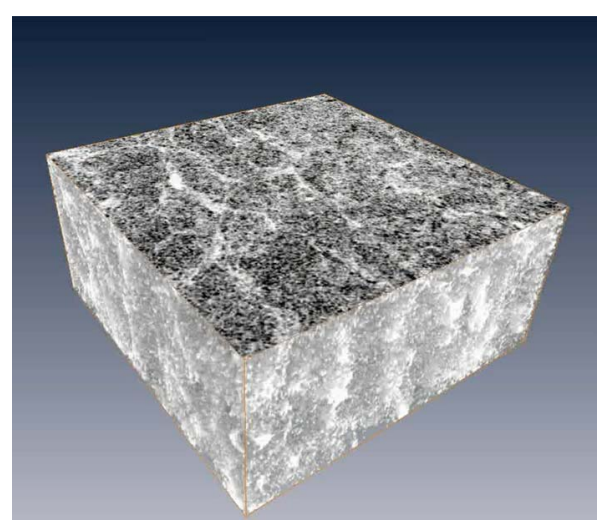

Video 3 Three-dimensional OCM reconstruction of human thyroid with follicular variant of papillary carcinoma. Normal follicles are absent from the specimen. Tightly packed microfollicles are the characteristic feature of the disease. Volume size is $400 \times 400 \times 180 \mu \mathrm{m}$ (QuickTime, 1.4 MB). [URL: http://dx.doi.org/10.1117/1.3306696.3]. 


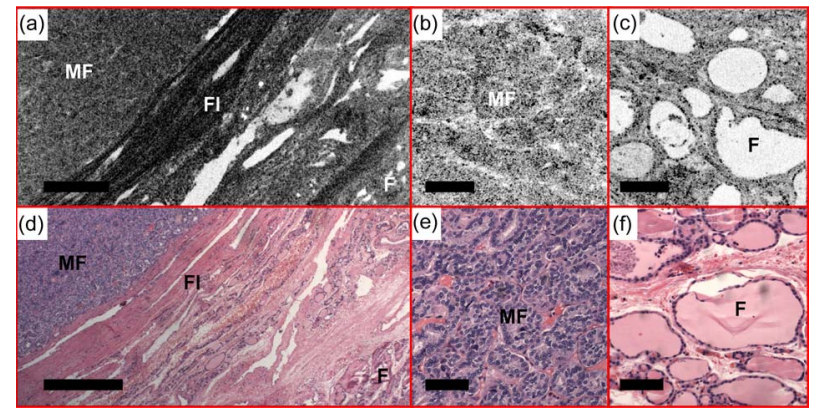

Fig. 15 (a) En face OCT shows the tumor interface with normal thyroid tissue in a case of papillary carcinoma, follicular variant. The tumor on the left side is clearly distinguished as densely packed microfollicles (MF), separated from the adjacent normal thyroid follicles $(\mathrm{F})$ at the lower right corner by a dense fibrous (FI) capsule. Details of microfollicles and normal follicles are shown in (b) and (c) the OCM images. The en face OCT and OCM images were obtained about 60 and $50 \mu \mathrm{m}$ below the tissue surface, respectively. (d), (e), and (f) are corresponding HE slides (4 and 20x, respectively). Scale bars, $500 \mu \mathrm{m}$ in (a) and (d) and $100 \mu \mathrm{m}$ in (b), (c), (e), and (f).

medullary carcinoma, normal thyroid follicles are absent. Sheets and nests of tumor cells are surrounded by dense fibrosis that can be clearly seen from the en face OCT image in Fig. 16. Details of the tumor nests can be visualized in the OCM image, matching the corresponding histology. Medullary carcinoma can have a variable histomorphologic appearance, so more examples will be sought in the future to better document this spectrum.

\section{Discussion}

Over the past three decades, a 2.4-fold increase in thyroid cancer incidence was observed in the United States. ${ }^{29}$ This was mainly due to an increased use of ultrasound for thyroid screening, which permits detection of nodules as small as 2 to $3 \mathrm{~mm}$. Several ultrasound features have been associated with an increased risk of thyroid cancer, including presence of calcifications, hypoechogenicity, irregular margins, solid composition, nodule shape, and intranodule vascularity. ${ }^{28}$ However, diagnostic accuracy of these criteria for malignancy is dependent on tumor size. ${ }^{30}$ Furthermore, considerable overlap between benign and malignant characteristics observed with ultrasound has been reported, ${ }^{31,32}$ and sensitivity and specificity of malignant nodule differentiation are variable. ${ }^{28,30,33-37}$ Current guidelines for management of thyroid nodules detected by ultrasound suggest performing FNA on nodules larger than $1 \mathrm{~cm}$ to make the diagnosis of a benign or malignant nodule., ${ }^{9,28}$ However, the sensitivity and specificity of thyroid FNA varies. $^{38}$

The OCT and OCM technologies presented in the current study have the potential to be a useful complementary technique for the evaluation of thyroid nodules. The axial and transverse resolutions of OCT and OCM are 1 to 2 orders of magnitude finer than the state of the art ultrasound technology. The integrated OCT and OCM system allows seamlessly switching between low and high magnifications in a way similar to traditional microscopy. The ability to visualize tissue morphology at multiple scales is very important for pathologists to differentiate clinical relevant features. As a re-

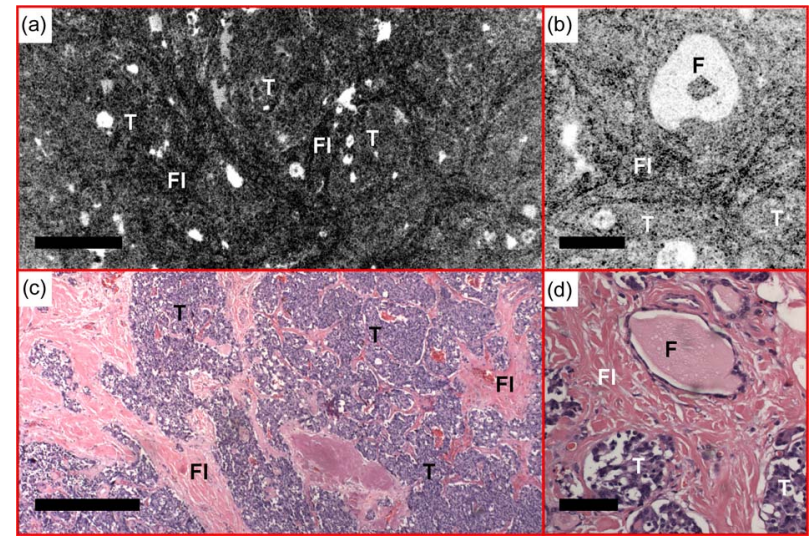

Fig. 16 Sheets and nests of tumor cells (T) are surrounded by fibrous bands (FI) in medullary carcinoma observed with (a) en face OCT and (b) OCM obtained about $50 \mu \mathrm{m}$ below the tissue surface. (c) and (d) are corresponding HE slides (4 and 20×, respectively). Scale bars, $500 \mu \mathrm{m}$ in (a) and (c), and $100 \mu \mathrm{m}$ in (b) and (d).

sult, characteristic architectural and cellular features from normal thyroid and benign and malignant thyroid diseases were successfully visualized at multiple resolution scales in excised specimens, without exogenous contrast agents or histological processing.

The ability of OCT and OCM to assess follicle shape and delineate growth patterns of thyroid tissues is valuable. The shapes of normal follicles are round to oval. Lesions containing macrofollicles are more likely to be benign, whereas nodules composed predominantly of microfollicles are more likely to be neoplastic. Features visualized in malignant diseases, such as the absence of normal follicles, and the presence of complex papillae, microfollicles, and sheet/nests of tumors, are approaching resolution at the cellular level. These intrinsic features form an image base that can be used to differentiate normal and benign nodules from malignancy using OCT and OCM.

One limitation of this study is the registration of images with histology. The method used in this study provides accurate registration between the region of pathology and histology. However, exact control of the histological plane is difficult, and combined with processing artifacts in histology, this makes precise one-to-one registration of histology to OCT/ OCM images challenging. Therefore, assessment of image and histological data is based on correspondence of feature information. This approach is consistent with methods used in other studies and addresses the objective of this study, enabling a correspondence to be established between features in OCT/OCM images and histology. Another limitation in the current study is the relatively small sample size, which prevents us from determining the sensitivity and specificity for assessment of thyroid malignancy. Prospective studies with a larger sample size and blinded image interpretation will be required to establish the clinical utility of OCT and OCM for thyroid neoplasia assessment.

Recently, significant efforts have been directed at miniaturizing OCT imaging probes for in vivo clinical applications, such as in gastroenterology ${ }^{39-41}$ and cardiology. ${ }^{42,43}$ Needlebased OCT imaging probes have also been demonstrated. ${ }^{44,45}$ Small diameter needle probes have been demonstrated with 
confocal and multiphoton microscopy. ${ }^{46-49}$ It is challenging to achieve a microscopy probe size comparable to the needles used for thyroid FNA. However, OCM uses both confocal and coherence detection to achieve cellular level transverse and axial resolution, even when a lower NA objective lens is used. This is an important advantage because it would facilitate the development of needle-based miniature OCM probes suitable for guiding FNA at cellular resolution.

The current OCT and OCM imaging system has excellent image resolution, but imaging speeds are limited and are not suited for in vivo applications. The recent development of Fourier/spectral domain and swept source OCT has yielded significant improvements in imaging speed. ${ }^{50,51}$ The development of Fourier domain mode-locked lasers enables speeds as high as 370,000 axial lines/s. ${ }^{52}$ This would enable imaging speeds of 275 cross-sectional frames per second for the volumetric OCT datasets described in this work. The field of view of 3-D OCT can be greatly enhanced with high speed imaging methods. A recent study demonstrated imaging of a $20 \times 8$ $\times 1.6-\mathrm{mm}^{3}$ volume within $20 \mathrm{~s}$ in the human gastrointestinal tract. ${ }^{53}$ Swept source OCM has also been demonstrated to achieve 3-D cellular resolution images at high speed. ${ }^{15}$ These technological advances would achieve imaging speeds sufficient for future clinical applications.

A needle-based OCT and OCM imaging device would provide complementary architectural information to ultrasound and FNA for in vivo evaluation of thyroid nodules. The combination of cytological and architectural information is expected to help clinicians make decisions for thyroid nodule management. In addition, OCT scans a larger field of view in real time, which could help locate higher yield areas for sampling with FNA. This could also potentially minimize the number of FNAs performed in benign nodules in the setting of multinodular colloid goiter. By excluding nodules with conclusive benign features up front, this will avoid potential cytologic false positive diagnoses and limit the number of nodules that require further evaluation, which would contribute to a reduction in cost for the workup of the incidentally detected thyroid nodule. One other potential future application involves the cytologic diagnosis of hypercellular follicular lesions on FNA. The diagnosis of follicular carcinoma cannot be made on cytologic specimens, because the definition of carcinoma requires histological examination of the entire capsule for capsular or vascular invasion. Patients with a cytologic diagnosis of hypercellular follicular lesion will usually undergo a hemithyroidectomy to rule out follicular carcinoma. A large majority of these nodules turn out to be follicular adenomas. The ability to distinguish between follicular adenoma and follicular carcinoma would be invaluable. OCT and OCM has the potential to assess capsular or vascular invasion in thyroid nodules with a predominant pattern of microfollicles. Further studies on excised tissue and in vivo trials will be needed to establish the clinical utility of OCT and OCM for thyroid nodule management.

\section{Acknowledgment}

This work was supported by NIH grants R01-CA75289-13 (Fujimoto and Connolly), Air Force Office of Scientific Research contract FA9550-07-1-0014 (Fujimoto), Medical Free Electron Laser Program contract FA9550-07-1-0101 (Fujimoto), and the MIT/CIMIT Medical Engineering Fellow- ship and Taiwan Merit Scholarship from the National Science Council of Taiwan (Tsai). The authors acknowledge useful discussions with Desmond C. Adler.

\section{References}

1. S. A. Hundahl, I. D. Fleming, A. M. Fremgen, and H. R. Menck, "A national cancer data base report on 53,856 cases of thyroid carcinoma treated in the US, 1985-1995," Cancer (N.Y.) 83, 2638-2648 (1998).

2. Cancer Facts and Figures, American Cancer Society, Atlanta, GA (2009).

3. P. W. Wiest, M. F. Hartshorne, P. D. Inskip, L. A. Crooks, B. S. Vela, R. J. Telepak, M. R. Williamson, R. Blumhardt, J. M. Bauman, and M. Tekkel, "Thyroid palpation versus high-resolution thyroid ultrasonography in the detection of nodules," J. Ultrasound Med. 17, 487496 (1998)

4. B. A. Carroll, "Asymptomatic thyroid-nodules-incidental sonographic detection," Am. J. Roentgenol. 138, 499-501 (1982).

5. A. Brander, P. Viikinkoski, J. Nickels, and L. Kivisaari, "Thyroidgland-ultrasound screening in a random adult-population," Radiology 181, 683-687 (1991).

6. J. N. Bruneton, C. Balumaestro, P. Y. Marcy, P. Melia, and M. Y. Mourou, "Very high-frequency (13 Mhz) ultrasonographic examination of the normal neck-detection of normal lymph-nodes and thyroid-nodules," J. Ultrasound Med. 13, 87-90 (1994).

7. B. Kneafsey, P. Gillen, and M. P. Brady, "Limitations of thyroid scanning in solitary thyroid-nodules," Ir J. Med. Sci. 163, 451-454 (1994).

8. S. I. Sherman, "Thyroid carcinoma," Lancet 361, 501-511 (2003).

9. B. R. Smith, D. S. Cooper, G. M. Doherty, B. R. Haugen, R. T. Kloos, S. L. Lee, S. J. Mandel, E. L. Mazzaferri, B. McIver, S. I. Sherman, and R. M. Tuttle, "Management guidelines for patients with thyroid nodules and differentiated thyroid cancer," Thyroid 16, 109142 (2006).

10. D. Huang, E. A. Swanson, C. P. Lin, J. S. Schuman, W. G. Stinson, W. Chang, M. R. Hee, T. Flotte, K. Gregory, C. A. Puliafito, and J. G. Fujimoto, "Optical coherence tomography," Science 254, 1178-1181 (1991).

11. J. A. Izatt, M. R. Hee, G. M. Owen, E. A. Swanson, and J. G. Fujimoto, "Optical coherence microscopy in scattering media," Opt. Lett. 19, 590-592 (1994).

12. A. D. Aguirre, P. Hsiung, T. H. Ko, I. Hartl, and J. G. Fujimoto, "High-resolution optical coherence microscopy for high-speed, in vivo cellular imaging," Opt. Lett. 28, 2064-2066 (2003).

13. W. Y. Oh, B. E. Bouma, N. Iftimia, S. H. Yun, R. Yelin, and G. J. Tearney, "Ultrahigh-resolution full-field optical coherence microscopy using InGaAs camera," Opt. Express 14, 726-735 (2006).

14. Y. Chen, S. W. Huang, A. D. Aguirre, and J. G. Fujimoto, "Highresolution line-scanning optical coherence microscopy," Opt. Lett. 32, 1971-1973 (2007).

15. S. W. Huang, A. D. Aguirre, R. A. Huber, D. C. Adler, and J. G. Fujimoto, "Swept source optical coherence microscopy using a Fourier domain mode-locked laser," Opt. Express 15, 6210-6217 (2007).

16. J. A. Izatt, M. D. Kulkarni, H. W. Wang, K. Kobayashi, and M. V. Sivak, Jr., "Optical coherence tomography and microscopy in gastrointestinal tissues," IEEE J. Sel. Top. Quantum Electron. 2, 10171028 (1996).

17. G. J. Tearney, M. E. Brezinski, J. F. Southern, B. E. Bouma, S. A. Boppart, and J. G. Fujimoto, "Optical biopsy in human gastrointestinal tissue using optical coherence tomography," Am. J. Gastroenterol. 92, 1800-1804 (1997).

18. G. J. Tearney, M. E. Brezinski, J. F. Southern, B. E. Bouma, S. A. Boppart, and J. G. Fujimoto, "Optical biopsy in human pancreatobiliary tissue using optical coherence tomography," Dig. Dis. Sci. 43, 1193-1199 (1998).

19. P. L. Hsiung, L. Pantanowitz, A. D. Aguirre, Y. Chen, D. Phatak, T. H. Ko, S. Bourquin, S. J. Schnitt, S. Raza, J. L. Connolly, H. Mashimo, and J. G. Fujimoto, "Ultrahigh-resolution and 3-dimensional optical coherence tomography ex vivo imaging of the large and small intestines," Gastrointest. Endosc. 62, 561-574 (2005).

20. Y. Chen, A. D. Aguirre, P. L. Hsiung, S. Desai, P. R. Herz, M. Pedrosa, Q. Huang, M. Figueiredo, S. W. Huang, A. Koski, J. M. Schmitt, J. G. Fujimoto, and H. Mashimo, "Ultrahigh resolution optical coherence tomography of Barrett's esophagus: preliminary de- 
scriptive clinical study correlating images with histology," Endoscopy 39, 599-605 (2007).

21. P. L. Hsiung, D. R. Phatak, Y. Chen, A. D. Aguirre, J. G. Fujimoto, and J. L. Connolly, "Benign and malignant lesion in the human breast depicted with ultrahigh resolution and dimensional optical coherence tomography," Radiology 244, 865-874 (2007).

22. S. A. Boppart, W. Luo, D. L. Marks, and K. W. Singletary, "Optical coherence tomography: feasibility for basic research and imageguided surgery of breast cancer," Breast Cancer Res. Treat 84, 85-97 (2004).

23. A. M. Zysk and S. A. Boppart, "Computational methods for analysis of human breast tumor tissue in optical coherence tomography images," J. Biomed. Opt. 11, 054015 (2006).

24. G. J. Tearney, M. E. Brezinski, J. F. Southern, B. E. Bouma, S. A. Boppart, and J. G. Fujimoto, "Optical biopsy in human urologic tissue using optical coherence tomography," J. Urol. (Baltimore) 157, 1915-1919 (1997).

25. A. V. D'Amico, M. Weinstein, X. Li, J. P. Richie, and J. Fujimoto, "Optical coherence tomography as a method for identifying benign and malignant microscopic structures in the prostate gland," Urology 55, 783-787 (2000).

26. L. Pantanowitz, P. L. Hsiung, T. H. Ko, K. Schneider, P. R. Herz, J. G. Fujimoto, S. Raza, and J. L. Connolly, "High-resolution imaging of the thyroid gland using optical coherence tomography," Head Neck 26, 425-434 (2004).

27. A. D. Aguirre, "Advances in optical coherence tomography and microscopy for endoscopic applications and functional neuroimaging," Ph.D. thesis, Dept. Electrical Eng. Computer Sci. Res. Lab. Electronics, Massachusetts Institute of Technology, Cambridge, MA (2008).

28. M. C. Frates, C. B. Benson, J. W. Charboneau, E. S. Cibas, O. H Clark, B. G. Coleman, J. J. Cronan, P. M. Doubilet, D. B. Evans, J. R. Goellner, I. D. Hay, B. S. Hertzberg, C. M. Intenzo, R. B. Jeffrey, J. E. Langer, P. R. Larsen, S. J. Mandel, W. D. Middleton, C. C. Reading, S. I. Sherman, and F. N. Tessier, "Management of thyroid nodules detected at US: Society of Radiologists in Ultrasound consensus conference statement," Radiology 238, 794-800 (2006).

29. L. Davies and H. G. Welch, "Increasing incidence of thyroid cancer in the United States, 1973-2002," JAMA, J. Am. Med. Assoc. 295, 2164-2167 (2006)

30. W. J. Moon, S. L. Jung, J. H. Lee, D. G. Na, J. H. Baek, Y. H. Lee, J. Kim, H. S. Kim, J. S. Byun, and D. H. Lee, "Benign and malignant thyroid nodules: US differentiation-multicenter retrospective study," Radiology 247, 762-770 (2008).

31. J. R. Wienke, W. K. Chong, J. R. Fielding, K. H. Zou, and C. A Mittelstaedt, "Sonographic features of benign thyroid nodulesinterobserver reliability and overlap with malignancy," J. Ultrasound Med. 22, 1027-1031 (2003).

32. J. D. Iannuccilli, J. J. Cronan, and J. M. Monchik, "Risk for malignancy of thyroid nodules as assessed by sonographic criteria-the need for biopsy," J. Ultrasound Med. 23, 1455-1464 (2004).

33. E. Papini, R. Guglielmi, A. Bianchini, A. Crescenzi, S. Taccogna, F. Nardi, C. Panunzi, R. Rinaldi, V. Toscano, and C. M. Pacella, "Risk of malignancy in nonpalpable thyroid nodules: Predictive value of ultrasound and color-Doppler features," J. Clin. Endocrinol. Metab. 87, 1941-1946 (2002).

34. M. L. C. Khoo, S. L. Asa, I. J. Witterick, and J. L. Freeman, "Thyroid calcification and its association with thyroid carcinoma," Head Neck 24, 651-655 (2002).

35. S. Peccin, J. A. S. de Castro, T. W. Furlanetto, A. P. A. Furtado, B. A. Brasil, and M. A. Czepielewski, "Ultrasonography: Is it useful in the diagnosis of cancer in thyroid nodules?," J. Endocrinol. Invest $\mathbf{2 5}$ 39-43 (2002).

36. E. K. Kim, C. S. Park, W. Y. Chung, K. K. Oh, D. I. Kim, J. T. Lee, and H. S. Yoo, "New sonographic criteria for recommending fineneedle aspiration biopsy of nonpalpable solid nodules of the thyroid,"
Am. J. Roentgenol. 178, 687-691 (2002).

37. M. C. Frates, C. B. Benson, P. M. Doubilet, E. S. Cibas, and E. Marqusee, "Can color Doppler sonography aid in the prediction of malignancy of thyroid nodules?" J. Ultrasound Med. 22, 127-131 (2003).

38. H. H. Wang, "Reporting thyroid fine-needle aspiration: literature review and a proposal," Diagn. Cytopathol 34, 67-76 (2006).

39. G. J. Tearney, M. E. Brezinski, B. E. Bouma, S. A. Boppart, C. Pitvis, J. F. Southern, and J. G. Fujimoto, "In vivo endoscopic optical biopsy with optical coherence tomography," Science 276, 2037-2039 (1997).

40. M. V. Sivak, Jr., K. Kobayashi, J. A. Izatt, A. M. Rollins, R. UngRunyawee, A. Chak, R. C. Wong, G. A. Isenberg, and J. Willis, "High-resolution endoscopic imaging of the GI tract using optical coherence tomography," Gastrointest. Endosc. 51(4), Pt 1, 474-479 (2000).

41. X. D. Li, S. A. Boppart, J. Van Dam, H. Mashimo, M. Mutinga, W. Drexler, M. Klein, C. Pitris, M. L. Krinsky, M. E. Brezinski, and J. G. Fujimoto, "Optical coherence tomography: advanced technology for the endoscopic imaging of Barrett's esophagus," Endoscopy 32, 921-930 (2000).

42. X. Li, T. H. Ko, and J. G. Fujimoto, "Intraluminal fiber-optic Doppler imaging catheter for structural and functional optical coherence tomography," Opt. Lett. 26, 1906-1908 (2001).

43. Y. Kawase, K. Hoshino, R. Yoneyama, J. McGregor, R. J. Hajjar, I. $\mathrm{K}$. Jang, and M. Hayase, "In vivo volumetric analysis of coronary stent using optical coherence tomography with a novel balloon occlusion-flushing catheter: a comparison with intravascular ultrasound," Ultrasound Med. Biol. 31, 1343-1349 (2005).

44. X. Li, C. Chudoba, T. Ko, C. Pitris, and J. G. Fujimoto, "Imaging needle for optical coherence tomography," Opt. Lett. 25, 1520-1522 (2000).

45. A. M. Zysk, D. L. Marks, D. Y. Liu, and S. A. Boppart, "Needlebased reflection refractometry of scattering samples using coherencegated detection," Opt. Express 15, 4787-4794 (2007).

46. V. Becker, T. Vercauteren, C. H. von Weyhern, C. Prinz, R. M Schmid, and A. Meining, "High-resolution miniprobe-based confocal microscopy in combination with video mosaicing (with video)," Gastrointest. Endosc. 66, 1001-1007 (2007).

47. B. A. Flusberg, E. D. Cocker, W. Piyawattanametha, J. C. Jung, E. L. M. Cheung, and M. J. Schnitzer, "Fiber-optic fluorescence imaging," Nat. Methods 2, 941-950 (2005)

48. J. N. Rogart, J. Nagata, C. S. Loeser, R. D. Roorda, H. Aslanian, M. E. Robert, W. R. Zipfel, and M. H. Nathanson, "Multiphoton imaging can be used for microscopic examination of intact human gastrointestinal mucosa ex vivo," Nat. Clin. Pract. Gastroenterol. Hepatol. 6, 95-101 (2008)

49. M. T. Myaing, D. J. MacDonald, and X. D. Li, "Fiber-optic scanning two-photon fluorescence endoscope," Opt. Lett. 31, 1076-1078 (2006).

50. S. H. Yun, G. J. Tearney, J. F. de Boer, N. Iftimia, and B. E. Bouma, "High-speed optical frequency-domain imaging," Opt. Express 11, 2953-2963 (2003).

51. R. Huber, M. Wojtkowski, and J. G. Fujimoto, "Fourier Domain Mode Locking (FDML): a new laser operating regime and applications for optical coherence tomography," Opt. Express 14, 32253237 (2006).

52. R. Huber, D. C. Adler, and J. G. Fujimoto, "Buffered Fourier domain mode locking: unidirectional swept laser sources for optical coherence tomography imaging at 370,000 lines/s," Opt. Lett. 31, 29752977 (2006).

53. D. C. Adler, C. Zhou, T. H. Tsai, J. Schmitt, Q. Huang, H. Mashimo, and J. G. Fujimoto, "Three-dimensional endomicroscopy of the human colon using optical coherence tomography," Opt. Express 17, 784-796 (2009). 